Case Report

Journal of Epilepsy Research pISSN 2233-6249 / eISSN 2233-6257

Received July 25, 2018

Revised December 4, 2018

Accepted December 24, 2018

Corresponding author:

Dae Lim Koo, MD, PhD

Department of Neurology, Seoul Metropolitan

Government Seoul National University

Boramae Medical Center, Seoul National

University College of Medicine, 20

Boramae-ro 5-gil, Dongjak-gu, Seoul 07061,

Korea

Tel. $+82-2-870-2473$

Fax. +82-2-831-0714

E-mail; koodaelim@gmail.com, koodaelim@naver.com

\title{
Acute Ischemic Stroke Mimicking Non-Convulsive Status Epilepticus
}

\author{
Ahwon Kim, MD, Sang Bin Hong, MD, Seonkyung Lee, MD, Heejung Mo, MD, \\ Dae Lim Koo, MD, PhD
}

Department of Neurology, Seoul Metropolitan Government Seoul National University Boramae Medical Center, Seoul National University College of Medicine, Seoul, Korea

\begin{abstract}
Status epilepticus and stroke are occasionally characterized by indistinguishable symptoms. Diffusion-weighted imaging (DWI) could not only help differentiating seizure from stroke, but also assist in localizing a focus of seizure and reflect resolution of seizure. We report a case of non-convulsive status epilepticus that presented as acute ischemic stroke, mimicking the latter's clinical manifestations. A 77-year-old right-handed man visited the emergency room with complaints of impaired awareness and fluency with right hemiparesis. Moreover, he presented with first-onset focal to bilateral tonic-clonic seizure with eyeball deviation to the right for 1 minute. Magnetic resonance imaging showed hyperintensity on DWI with a reduced apparent diffusion coefficient (ADC) value in the left pulvinar and left temporo-occipital areas. However, eyeball deviation to the right was not matched with left hemispheric stroke. Electroencephalography (EEG) confirmed ictal discharges in the left temporal area, which were compatible with impaired awareness, ictal aphasia, and eyeball deviation to the right. In cases of conflicting results from neurological examination and DWI/ADC findings, clinicians should consider employing EEG for diagnosing and treating non-convulsive status epilepticus. (2018;8:90-92)
\end{abstract}

Key words: Status epilepticus, Stroke, Electroencephalography, Magnetic resonance imaging

\section{Introduction}

Status epilepticus and acute ischemic stroke are the most common emergency neurological disorders. Hyperintensity on diffusion-weighted magnetic resonance imaging (DWI) has been useful in localizing a focus of both conditions. ${ }^{1}$ Here, we report a case of non-convulsive status epilepticus that presented as acute ischemic stroke, mimicking the latter's clinical manifestations.

\section{Case}

A 77-year-old right-handed man visited emergency room (ER) of Boramae Medical Center with complaints of impaired awareness and fluency that had presented 2.5 hours ago. Neurological examination revealed alert mental status, impaired verbal fluency, and decreased spontaneous movements in the right upper and lower extremities. The patient had a past history of persistent atrial fibrillation, chronic obstructive pulmonary disease, and non-alcoholic fatty liver disease, for which he takes apixaban and bronchodilators. No history of seizures, trauma, and stroke was identified. He denied probability of sleep deprivation, alcohol drinking, recent infection, and poor oral intake. During the ER stay, he developed first-onset focal to bilateral tonic-clonic seizure, with eyeball deviation to the right for 1 minute. Convulsive movement and eyeball deviation were managed by an injection of lorazepam. Initial arterial blood gas analysis and routine laboratory parameters, including complete blood count, renal function, liver function, and lactate level, were within the reference range, except for mild elevated C-reactive protein $(2.2 \mathrm{mg} / \mathrm{dL})$. DWI showed high signal intensities restricted to the left pulvinar and temporo-occipital regions (Fig. 1A), in which the apparent diffusion coefficient (ADC) decreased (Fig. 1B). Magnetic resonance (MR) angiography showed total occlusion of the right internal carotid artery, and severe stenosis of both anterior cerebral arteries (Fig. 1C). A perfusion study highlighted a delayed value of time to peak in territories of the right middle cerebral artery and both anterior cerebral arteries (Fig. 1D). Global aphasia and reduced right limb movements persisted and were not clearly explained on DWI or the perfusion map. DWI abnormalities could be considered seizure-related changes because eyeball deviation to the right 

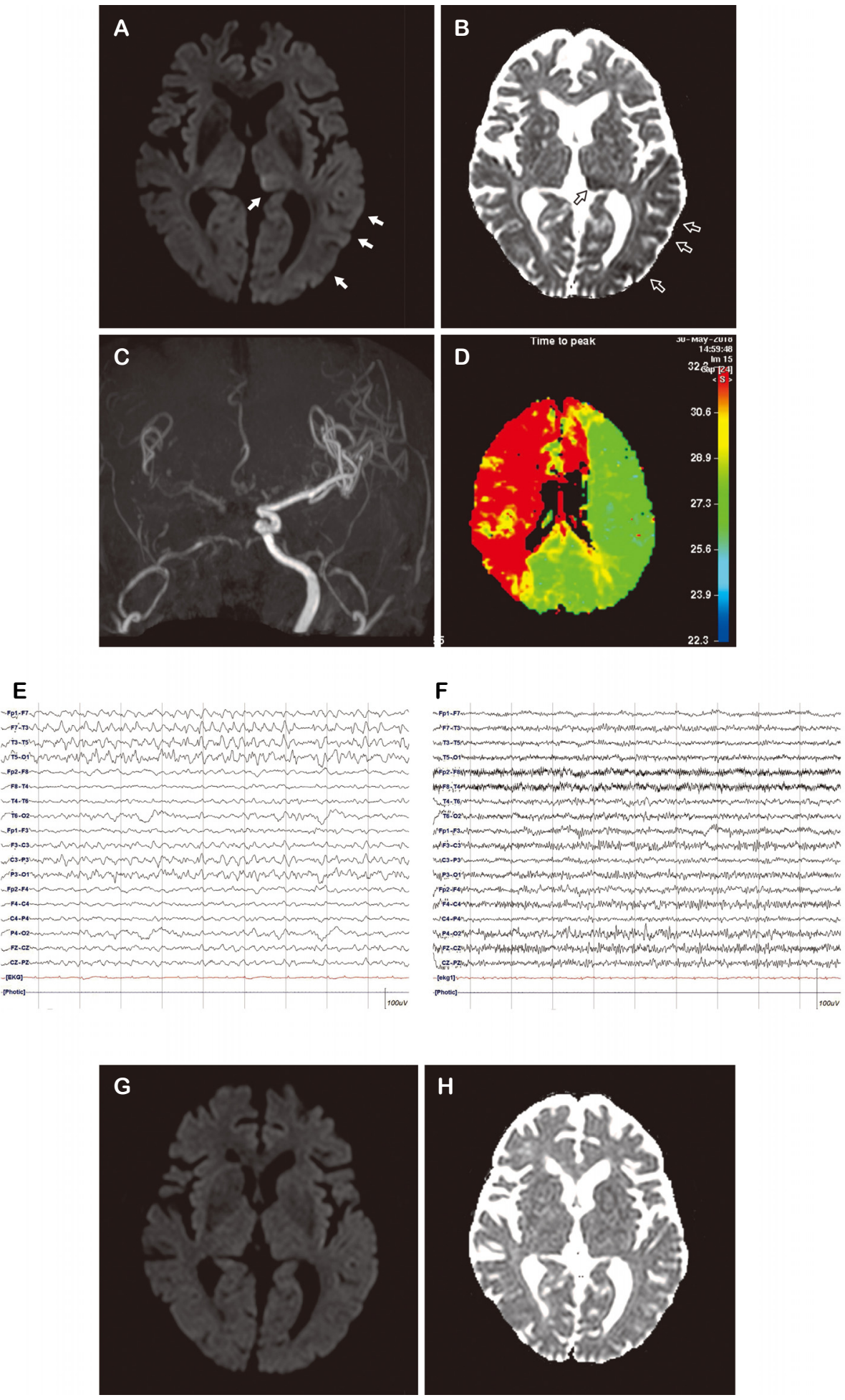

Figure 1. Results of MR image, MR angiography, perfusion map, and EEG. (A-E) Findings before the administration of antiepileptic drugs. (F-H) Results after $\geq 24$ hours of no seizure event. (A, B) Initial DWI shows high signal intensities on the left pulvinar and temporo-occipital areas (closed arrows), in which the $A D C$ is decreased (open arrows). (C) MR angiography reveals total occlusion of the right proximal internal carotid artery. (D) Perfusion image represents the delayed value of time to peak at the territory of the right middle cerebral artery and both anterior cerebral arteries. (E) Initial EEG indicates semirhythmic theta-to-delta activities lateralized to the left temporal region. (F) After gaining control of the non-convulsive status epilepticus, EEG showed no epileptiform discharges. $(G, H)$ Follow-up MR images present the normalized DWI and ADC values. MR, magnetic resonance; EEG, electroencephalography; DWI, diffusion-weighted imaging; $A D C$, apparent diffusion coefficient. 
implies a contralateral value from the left hemispheric ictal focus; decreased verbal fluency might be a phenomenon of ictal aphasia. Electroencephalography (EEG) was performed with a clinical impression of non-convulsive status epilepticus. Baseline EEG showed semirhythmic theta-to-delta activities lateralized to the left temporal region (Fig. 1E). Continuous EEG monitoring and administration of phenytoin, levetiracetam, and phenobarbital were prescribed. To exclude any infection of the central nervous system, a cerebrospinal fluid study was conducted, which showed normal white blood cell, protein, and glucose levels. After 2 days of symptom onset, verbal fluency and motor power completely recovered and no epileptiform discharges were observed on EEG (Fig. 1F). A follow-up MR scan revealed a resolved state of diffusion restriction and low ADC value in the left pulvinar and left temporo-occipital area (Fig. 1G, H).

\section{Discussion}

The present case presented impaired awareness, decreased verbal fluency, and right hemiparesis, which raised a diagnostic possibility of acute stroke in the left hemisphere. However, eyeball deviation to the right did not correspond to left hemispheric stroke. Furthermore, findings on DWI and MR angiography were not correlated with these symptoms and signs. EEG confirmed ictal discharges in the left temporal area, which was compatible with impaired awareness, ictal aphasia, and eyeball deviation to the right. MR findings, including hyperintensity on DWI with low ADC value from hemodynamic and tissue changes, can support the evidence of ictal or peri-ictal phases in non-convulsive status epilepticus. ${ }^{2,3}$ The most common location of DWI abnormalities associated with ictal activity is the hippocampus and pulvinar of the thalamus. ${ }^{4}$ Follow-up DWI revealed a resolved state of hyperintensity on DWI and low signals on $A D C$, which corresponded to both clinical improvement of non-convulsive status epilepticus and absence of epileptiform discharges on EEG. In our case, eyeball deviation to the right may be a sign of either acute ischemic stroke in the right middle cerebral artery territory or ictal activity of the left hemisphere. If the ictal discharges continued in the language-dominant hemisphere, speech arrest could be an important localizing sign. A combination of DWI and EEG analysis can provide diagnostic clues for seizure localization and propagation, particularly in non-convulsive status epilepticus. A previous study reported that EEG abnormalities correlated with lateralization of DWI abnormalities in $81.5 \%$ of cases. ${ }^{4}$ Our case provides further evidence that the combination of acute changes and normalization of the DWI/ADC value and EEG findings are useful, particularly in patients with a clinical impression of both acute ischemic stroke and non-convulsive status epilepticus. In cases of conflicting results from neurological examination and DWI/ADC findings, clinicians should consider employing EEG to diagnose and treat non-convulsive status epilepticus.

\section{Conflicts of Interest}

We have no affiliations with or involvement in any organization or entity with any financial interest, or non-financial interest. The authors report no disclosures.

\section{References}

1. Chu K, Kang DW, Kim JY, Chang KH, Lee SK. Diffusion-weighted magnetic resonance imaging in nonconvulsive status epilepticus. Arch Neuro/ 2001; 58:993-8.

2. Szabo K, Poepel A, Pohlmann-Eden B, et al. Diffusion-weighted and perfusion MRI demonstrates parenchymal changes in complex partial status epilepticus. Brain 2005;128(Pt 6):1369-76.

3. Flacke $S$, Wüllner $U$, Keller $E$, Hamzei $F$, Urbach H. Reversible changes in echo planar perfusion- and diffusion-weighted MRI in status epilepticus. Neuroradiology 2000;42:92-5.

4. Chatzikonstantinou A, Gass A, Förster A, Hennerici MG, Szabo K. Features of acute DWI abnormalities related to status epilepticus. Epilepsy Res 2011;97:45-51. 